\title{
PROPOSIÇÃO E TESTE DE UMA ESCALA DE DINAMISMO, COMPLEXIDADE E MUNIFICÊNCIA AMBIENTAL.
}

\section{RESUMO}

Considerando o interesse que a área de estratégia tem sobre o ambiente e seu impacto na organização, este trabalho aborda a questão sobre como se pode mensurar este ambiente. Objetiva construir e validar escalas de mensuração das dimensões de dinamismo, complexidade e munificência do ambiente percebido pelo gestor. As escalas foram construídas com base na teoria revisada, aplicadas a uma amostra de 170 gestores de hotéis brasileiros e validadas com a utilização de técnica de análise fatorial, com sua confiabilidade testada através da análise das comunalidades, da variância extraída pelo fator, do teste de esfericidade de Bartlet, do teste Alfa de Cronbach, da análise de correlações e do método split half. As escalas validadas, após rotação Varimax, apresentaram-se ortogonais entre si, demonstrando independência. O construto complexidade foi formado pela dificuldade de obtenção de informações e de entendimento do ambiente, pela dispersão da informação no ambiente e pela sua imprevisibilidade, conforme previsto anteriormente por Cannon e St. John (2007). Dinamismo foi validado como formado pela variabilidade nos diversos componentes do ambiente competitivo no qual a firma está inserida, conforme preconizado por Lumpkin e Dess (2001), enquanto o construto munificência refletiu a existência de recursos disponíveis, a ampliação da disponibilidade de recursos, a oferta de mais oportunidades do que ameaças aos competidores e o crescimento do mercado, conforme mencionado anteriormente por Castrogiovanni (1991).

Palavras-chave: Ambiente Organizacional; Dinamismo; Complexidade; Munificência; Escalas de Mensuração.

\section{PROPOSITION AND TEST OF THE ONE SCALE OF THE ENVIRONMENTAL DYNAMISM, COMPLEXITY AND MUNIFICENCE.}

\begin{abstract}
Considering the concernment that the strategy area has about the environment and its impact on organization, this paper discuss about the environment mensuration. Its aim is build and validate scales of mensuration of the perceived dynamism, complexity and munificence dimensions. The scales were built on the revised theory, applied to a sample of 170 Brazilian hotel managers and validate under the factorial analysis with tests of reliability trough communalities, variance explained, Bartlet's sphericity test, Cronbach's Alpha, correlation analysis, and split half method. After the Varimax rotation, the constructs showed independent and orthogonal among them. The complexity construct was built by gaining information and environment understanding difficult, by information dispersion and by its unpredictability. Dynamism was validate as the variability in the components of the competitive environments, while the munificence construct reflect the availability of existing resources, the growing of the available resources, the existing of more opportunities than threats, and the growing of the market.
\end{abstract}

Keywords: Organizational Environment; Dynamism; Complexity; Munificence; Mensuration Scales. 
Proposição e Teste de uma Escala de Dinamismo, Complexidade e Munificência Ambiental.

PROPUESTA Y PRUEBAS DE UN RANGO DINÁMICO, COMPLEJO Y MUNIFICENCIA AMBIENTAL.

\section{RESUMEN}

Considerando el interés que el área de la estrategia tiene en el medio ambiente y su impacto en la organización, este documento aborda la cuestión de cómo podemos medir este entorno empresarial. Tiene como objetivo desarrollar y validar escalas de medición de las dimensiones de dinamismo, complejidad y munificencia del ambiente percibido por el gerente. Las escalas se construyeron sobre la base de la teoría revisada, aplicado a una muestra de 170 gerentes de hoteles brasileños y validado con el uso de análisis factorial, con su fiabilidad probado mediante el análisis de las similitudes, la varianza extraída por el factor, el ensayo esfericidad Bartlet, prueba alfa de Cronbach, el análisis de correlación y el método de split half. Las escalas validadas después de la rotación Varimax, se presenta ortogonales entre sí, mostrando la independencia. La construcción de la complejidad fue formada por la dificultad de obtener información y comprensión del medio ambiente, la dispersión de la información en el medio ambiente y su imprevisibilidad, como se predijo anteriormente por Cannon y St. John (2007). Dinamismo fue validado como formado por la variabilidad en los diversos componentes del entorno competitivo en el que opera la empresa, según lo recomendado por Lumpkin y Dess (2001), mientras que la construcción de la munificencia refleja la existencia de los recursos disponibles, la expansión de la disponibilidad de recursos, el suministro más oportunidades que amenazas para los competidores y el crecimiento del mercado, como anteriormente mencionadas por Castrogiovanni (1991).

Palabras clave: Ambiente Organizacional; Dinamismo; Complejidad; Munificencia; Escalas de Medida.

\footnotetext{
${ }^{1}$ Doutor em Administração e Turismo pela Universidade do Vale do Itajaí - UNIVALI. Professor do Mestrado em Desenvolvimento Regional da Universidade do Contestado - UnC. Brasil. E-mail: carloseduardo@ unc.br

${ }^{2}$ Doutor em Engenharia de Produção pela Universidade Federal de Santa Catarina - UFSC. Professor da Universidade do Vale do Itajaí - UNIVALI. Brasil. E-mail: rossetto@univali.br
} 


\section{INTRODUÇÃO}

A área de estratégia tem se preocupado intensamente com o ambiente no qual a organização está inserida, ambiente este que tem sido confirmado como fonte de influências tanto para a atuação das firmas, quanto para os resultados que as firmas conseguem obter (Qu, Pinsonneault \& Oh, 2011; Xue, Ray \& Sambamurthy, 2012). Por outro lado, há ainda discussões sobre quais dimensões deste ambiente são relevantes para o estudo na área (Lukas, Tan \& Hult, 2001), e principalmente, como se podem mensurar estas dimensões (Porto, Brito, Silva, Bataglia \& Brito, 2009).

Alguns estudos tem se proposto a mensurar o ambiente através de dados objetivos obtidos de fontes secundárias, como Dess e Beard (1984) e (Porto et al., 2009), enquanto outros trabalhos defendem a utilização de dados subjetivos (Luo, Tan \& O'Connor, 2001; Bertucci, 2005). Entre os estudos com dados subjetivos as escalas de percepção em sua maioria são baseadas em segmentos do ambiente como Tan e Litschert (1994), Luo et al. (2001) e Teixeira, Rossetto e Carvalho (2009). Assim, o objetivo deste trabalho é construir e avaliar a validade e confiabilidade de uma escala de mensuração do dinamismo, da complexidade e da munificência do ambiente, baseadas no conhecimento acumulado na área, e que possam ser utilizadas em trabalhos realizados a partir da percepção do gestor. A validação de escalas para a mensuração das dimensões do ambiente traz contribuição relevante e inovadora à área da estratégia ao possibilitar meios válidos de mensuração destas dimensões o que possibilita a utilização de metodologias de testes de relacionamento entre o ambiente e outros construtos.

\section{AMBIENTE ORGANIZACIONAL}

Entre os estudos da área de estratégia preocupados com o ambiente podem ser encontrados aqueles que procuram identificar o ambiente objetivo ou real das organizações e aqueles que estão interessados no ambiente subjetivo ou percebido, ou seja, aquele interpretado pelos atores organizacionais (Rossetto et al., 2012). Na visão de Sharfman e Dean (1991) este tem sido o debate central sobre o ambiente nos estudos organizacionais. Entre aqueles que têm adotado a opção pelo ambiente objetivo, este tem sido mensurado através de indicadores setoriais, como os propostos por Dess e Beard (1984), e tem sido utilizados para comparar os ambientes entre diversos setores empresariais, como a aplicação de Porto et al. (2009).

Porém, é crescente o número de estudos que considera o ambiente percebido como fundamental para o entendimento das ações estratégicas das firmas. Neste sentido, a abordagem da escolha estratégica considera o ambiente como representado e interpretado pelo gestor, atribuindo-se a esse maior poder de influência sobre o ambiente e maior grau de discricionariedade em suas decisões (Child, 1972; Rossetto et al., 2012; Simsek, Veiga \& Lubatkin, 2007).

Neste sentido, a atenção dos estudos tem se deslocado do ambiente objetivo que rodeia a organização para o ambiente subjetivo, percebido pelos seus gestores (Duncan, 1972; Milliken, 1987), considerando-se a elevada importância atribuída a esse ambiente percebido pelos gestores no processo de tomada de decisões (Luo et al., 2001), bem como o potencial de problemas de validade da utilização de dados secundários como proxies (Olsen, 2004).

Outra questão relacionada aos estudos sobre o ambiente é a definição de qual nível de ambiente se está estudando. Osborn e Hunt (1974) definiram o ambiente como possuindo três níveis, macro, agregado e ambiente de tarefa. Preocupado com o problema de excesso de abstração nos estudos em sobre ambiente, Castrogiovanni (1991) foi além propondo a existência de cinco níveis ambientais, o macro ambiente, o ambiente agregado, o ambiente de tarefa, os subambientes, e o nível das reservas de recursos.

No nível do ambiente de tarefa os diversos subambientes de uma organização são vistos em combinação. Segundo Osborn e Hunt (1974) para cada organização este ambiente consiste de todas aquelas organizações com as quais ela precisa interagir para crescer e sobreviver, incluindo clientes, fornecedores, entre outros. Tan e Litschert (1994) propuseram o uso de oito fatores do ambiente, fornecedores, tecnológicos, sociais-culturais, internacionais, regulatórios, competidores, econômicos, e consumidores. Luo et al. (2001) comentam sobre aquilo que chamam de fontes de impacto ambiental, sendo caracterizadas como os segmentos do ambiente, incluídos os competidores, fornecedores, consumidores e os aspectos regulatórios. Estes segmentos estão ancorados nas dimensões do ambiente industrial, fornecedores, consumidores e competidores (Miller, 1992; Porter, 1999) e são acrescidos dos aspectos regulatórios.

Castrogiovanni (1991) sugere que os pesquisadores deveriam examinar o ambiente no nível mais apropriado para a questão de pesquisa específica, o que não impede que sejam também analisados níveis adjacentes do ambiente que possam ser relevantes para o estudo.

Como o objetivo deste trabalho está relacionado à validação de escala própria para a mensuração e comparação do ambiente percebido pelos gestores, a opção é pelo estudo do ambiente de tarefa ou ambiente operacional. Para Castrogiovanni (1991) os estudos neste nível devem focar nas decisões, ações, resultados, e características da organização como um todo para explicar similaridades e diferenças entre organizações. 
Proposição e Teste de uma Escala de Dinamismo, Complexidade e Munificência Ambiental.

\subsection{Dimensões de Estudo do Ambiente}

Para Lukas et al. (2001) há duas perspectivas distintas e complementares em relação ao ambiente, a primeira que considera o ambiente como fonte de informação, e a segunda que considera o ambiente como fonte de recursos. Dentro da primeira visão estão os estudos sobre a incerteza, como os de Duncan (1972), Milliken (1987), Magueiro (2010) e Gardelin (2011), enquanto dentro da segunda visão estão os estudos da teoria da dependência de recursos (Pfeffer \& Salancik, 1978), como o estudo de Jogaratnan (2002).

No início da década de 70, Duncan (1972) propôs uma tipologia para classificação dos ambientes percebidos pelos gestores. O trabalho considera duas dimensões fundamentais, que em conjunto darão o grau de incerteza no processo decisório da organização, uma dimensão de complexidade (simplex-complexo) e uma dimensão de dinamismo (estático-dinâmico). Trabalhos recentes utilizando a tipologia de Duncan (1972) incluindo Teixeira, Rossetto e Carvalho (2009) e Rossetto et al. (2012) têm encontrado forte associação entre as duas dimensões, ou seja, quando o gestor percebe o ambiente como dinâmico, tende também a o perceber como complexo.

Dess e Beard (1984) identificaram três dimensões importantes no ambiente, a complexidade, o dinamismo, e a munificência, a mesma conclusão encontrada por Sharfman e Dean Jr. (1991) que pressupõem que o estudo do ambiente passa por uma discussão sobre (a) o grau no qual o número e sofisticação dos elementos no ambiente tornam o entendimento mais difícil, (b) a estabilidade/previsibilidade de um ambiente, e (c) o nível de recursos disponíveis em um ambiente, relativo ao número de firmas competindo por estes recursos. Lumpkin e Dess (2001) consideram que o dinamismo e a complexidade refletem o grau de incerteza encontrado pelas organizações enquanto a munificência reflete o grau de dependência de uma organização em relação a seu ambiente. De maneira semelhante Luo et al. (2001) defendem a existência de três dimensões que caracterizam a natureza do impacto do ambiente, a complexidade, o dinamismo e a hostilidade, sendo que os autores reconhecem esta última como uma função inversa da munificência.

Diversos trabalhos recentes, que se propõem a analisar o ambiente de tarefa têm utilizado as dimensões dinamismo, complexidade e munificência propostas por Dess e Beard, como por exemplo, Harris (2004), Andrews (2009) e Porto et al. (2009).

A Figura 1 apresenta uma síntese das dimensões de estudo do ambiente propostas na literatura revisada para este trabalho. Considerando a recorrência das dimensões dinamismo, complexidade e munificência ambientais, este trabalho fez a opção por estas mesmas dimensões.

\begin{tabular}{|l|l|}
\hline \multicolumn{1}{|c|}{ ESTUDO } & \multicolumn{1}{c|}{ DIMENSÕES PROPOSTAS } \\
\hline Dess e Beard (1984) & Dinamismo, complexidade e munificência \\
\hline Scharfmann e Dean (1991) & Dinamismo, complexidade e munificência \\
\hline Lumpkin e Dess (2001) & Dinamismo e complexidade \\
\hline Luo, Tan e O'Connor (2001) & Dinamismo, complexidade e hostilidade \\
\hline Lukas, Tan e Hult (2001) & Dinamismo, complexidade e hostilidade \\
\hline Harris (2004) & Dinamismo, complexidade e munificência \\
\hline Andretti (2008) & Dinamismo e complexidade \\
\hline Andrews (2009) & Dinamismo, complexidade e munificência \\
\hline Porto et al. (2009) & Dinamismo, complexidade e munificência \\
\hline Gardelin (2010) & Munificência e incerteza \\
\hline Malgueiro (2011) & Dinamismo e complexidade \\
\hline Andrews e Johansen (2012) & Munificência, complexidade e dinamismo \\
\hline
\end{tabular}

Figura 1 - Dimensões do ambiente

\subsubsection{Complexidade ambiental}

O estudo da complexidade ambiental está entre as abordagens vistas por Lukas et al. (2001) que vêem o ambiente como fonte de informações para a firma. Já, Khandwalla (1972), assim como Duncan (1972) afirmavam que a complexidade é uma das dimensões que formam a incerteza ambiental, junto com o dinamismo. A dimensão simples-complexo de Duncan (1972) está assentada no número de fatores a serem considerados pelo gestor no seu processo decisório, bem como em quão similares ou diferentes entre si são estes fatores. Quanto maior o número de fatores, e quanto maior a diferença entre uns e outros, maior será a complexidade do ambiente.

Khandwalla (1972) considerava a heterogeneidade como uma dimensão distinta do ambiente. Porém, diversos trabalhos que se propõem a medir a complexidade tem incluído a heterogeneidade como seu componente. Luo et al. (2001) inclui a heterogeneidade como componente da complexidade, que foi definida como a média da heterogeneidade e diversidade de cada segmento. De maneira similar o trabalho de Porto et al. (2009) definem complexidade como a heterogeneidade ou amplitude das atividades essenciais de uma organização, bem como o grau de 
Proposição e Teste de uma Escala de Dinamismo, Complexidade e Munificência Ambiental.

sofisticação requerido para seu desempenho, envolvendo muitas interações para a tomada de decisão, e elevado grau de abstração na gestão.

Muitos trabalhos têm seguido a proposta de Dess e Beard (1984), os quais propuseram duas subdimensões da complexidade, a heterogeneidade e a dispersão. Sharfman e Dean Jr. (1991) consideram que a discussão da complexidade deve girar em torno do grau no qual o número e sofisticação dos elementos no ambiente tornam o seu entendimento mais difícil. Já, Lukas et al. (2001) entendem a complexidade como a previsibilidade do ambiente competitivo. Outro trabalho que levanta o número de questões diferentes ocorrendo simultaneamente, como definidor da complexidade é o de Harrington e Kendall (2007), para os quais o crescimento do número de questões interrelacionadas acontecendo em determinado contexto aumenta a dificuldade para os gestores interpretarem o impacto destas questões no seu negócio.

Recentemente, Cannon e St. John (2007) revisaram as propostas de Dess e Beard (1984) e de Sharfman e Dean (1991), em trabalho em que apresentam convergência conceitual com ambos os trabalhos, porém sugerem um novo leque de indicadores para mensuração, incluindo número de funcionários por organização, número de recursos ou entradas, número de diferentes grupos de clientes, número de produtos de cada firma na indústria, número de instituições com as quais as firmas interagem, montante de conhecimento científico necessário para as firmas interagirem na sua indústria, fragmentação do cenário competitivo, nível de complexidade tecnológica mensurado pelo percentual de engenheiros e cientistas empregados na indústria, complexidade dos processos relativa ao estágio do ciclo de vida da indústria e ao nível de intensidade de capital, e concentração geográfica.

Conceitualmente, este trabalho concorda com Sharfman e Dean (1991) e com Cannon e St. John (2007) quanto aos elementos que compõem a complexidade, sendo esta vista como formada pela heterogeneidade do segmento, pela sua dispersão, bem como pela dificuldade de obtenção de informações e de entendimento da evolução do segmento. Como estes trabalhos propõem operacionalização da mensuração através de dados secundários, neste trabalho foi construída e validada uma escala de mensuração da complexidade, conforme apresentado na metodologia. Como o próprio trabalho de Cannon e St. John (2007) explica, a utilização de dados objetivos é interessante para a comparação entre indústrias, porém o objetivo deste trabalho é a comparação entre a percepção de cada gestor.

\subsubsection{Dinamismo ambiental}

De maneira geral, as propostas conceituais relacionadas ao dinamismo ambiental se baseiam no ritmo de mudanças observado nos elementos do ambiente. Thompson (1967) já descrevia o dinamismo ambiental como as mudanças imprevisíveis dos elementos do ambiente. A dimensão estático-dinâmico de Duncan (1972) se refere à estabilidade dos fatores considerados no processo de tomada de decisão.

Tanto o trabalho de Duncan (1972) quanto o trabalho de Khandwalla (1972) consideram o dinamismo como sendo uma das dimensões que forma a incerteza, junto com a dimensão complexidade. Esta relação do dinamismo com a incerteza é reforçada no trabalho de Lumpkin e Dess (2001) que entende dinamismo como a taxa de mudanças imprevistas no ambiente de uma firma, estando relacionado à incerteza que diminui a habilidade dos gestores predizerem eventos futuros e seu impacto na organização. Esta última definição está de acordo com o entendimento de Lukas et al. (2001) de que o dinamismo se refere às mudanças no ambiente competitivo. Para estes últimos, o dinamismo estaria dentro da perspectiva do ambiente como fonte de informação.

No trabalho de Luo et al. (2001) dinamismo foi visto como a média da variabilidade e imprevisibilidade, e no de Harrington e Kendall (2007) como o nível de volatilidade no ambiente competitivo. O trabalho de Davis, Eisenhardt \& Bingham (2009) modelou o dinamismo ambiental como um construto multidimensional composto por velocidade, complexidade, ambiguidade e imprevisibilidade. Algumas destas dimensões são aqui tratadas como componentes do construto complexidade.

Mesmo havendo coerência teórica entre os pesquisadores sobre o que representa o dinamismo, pesquisadores como Boyd, Dess e Rasheed (1993) têm identificado diferenças na mensuração do construto entre estudos baseados em dados secundários com os baseados na percepção dos atores organizacionais. Porém, Harrington e Kendall (2005) testaram a operacionalização do dinamismo com dados secundários e dados percebidos, concluindo que quando são operacionalizados sobre o mesmo domínio, os dois tipos de medidas geram resultados convergentes.

Este trabalho adota a abordagem proposta por Lumpkin e Dess (2001) sob a qual o dinamismo é visto como a taxa de mudanças imprevistas no ambiente de uma firma. Para fins operacionais foi desenvolvida uma nova escala, que é apresentada na metodologia.

\subsubsection{Munificência ambiental}

Apesar de ser reconhecida por muitos estudiosos da área de estratégia como uma importante dimensão do ambiente (Dess \& Beard, 1984; YasaiArdekani, 1989; Sharfman \& Dean, 1991). A munificência está relacionada à visão do ambiente como fonte de recursos para a firma na definição de Lukas et al. (2001). Comparativamente ao dinamismo e à complexidade, a munificência ambiental tem recebido menor atenção (Harrington \& Kendall, 2005).

O termo surgiu nos estudos em estratégia a partir dos anos 1970. Pfeffer e Salancik (1978), sob a perspectiva da dependência de recursos, consideram 
Proposição e Teste de uma Escala de Dinamismo, Complexidade e Munificência Ambiental.

munificência a disponibilidade e abundância de recursos críticos no ambiente em que a firma está inserida. Castrogiovanni (1991) estuda o construto da munificência ambiental, propondo que a sua constituição seja mensurada através do crescimento ou declínio do mercado, da concentração de ameaças ou oportunidades, e da capacidade disponível no ambiente, esta última vista como os recursos disponíveis.

Lukas et al. (2001) conectando seu conceito de hostilidade ambiental à dependência de recursos a interpretam como o impacto do ambiente competitivo sobre a firma. Da mesma forma, Lumpkin e Dess (2001) utilizaram o termo hostilidade considerando-o como o oposto a munificência, seguindo Dess e Beard (1984) que a definem como escassez e intensidade na competição por recursos. Outro trabalho que utiliza hostilidade como o oposto de munificência é o de Luo et al. (2001), que vê a hostilidade como a média da dissuasão e criticidade dos recursos controlados pelos diferentes stakeholders.

Apesar de não utilizar os termos munificência ou hostilidade, o trabalho de Salazar \& Peláez (2011) estuda ambientes com restrições de recursos e de capacidades e que apresentam baixos níveis de demanda para novos produtos e serviços, fatores tradicionalmente tidos como componentes da munificência.

Diversas são as possíveis abordagens empíricas do conceito de munificência, desde as baseadas em dados secundários, como a do trabalho de YasaiArdekani (1989) que a operacionalizou através de dados secundários da demanda na indústria, até aquelas baseadas na percepção dos gestores, como o trabalho de Jogaratnan, Tse \& Olsen (1999) que operacionalizou a munificência com uma escala que mensurou a satisfação dos gestores com relação aos três componentes propostos por Castrogiovanni (1991).
Neste trabalho o conceito de munificência adotado é o proposto por Castrogiovanni (1991) composto pela capacidade do ambiente, pelo crescimento ou declínio das capacidades do ambiente, e pelo nível de oportunidades ou ameaças existente no ambiente. Para operacionalizá-lo foi criada uma escala que é apresentada na metodologia.

\section{METODOLOGIA}

Os trabalhos que se utilizam de coleta de dados em survey, em ciências sociais, têm utilizado muito a coleta de dados em escalas, e muitas vezes estes dados são tratados como se fossem dados contínuos o que pode comprometer a robustez dos testes estatísticos realizados por apresentarem problemas de normalidade (Finney \& Distefano, 2006). Neste sentido Bollen (1989) já havia afirmado que escalas formadas por um número maior de categorias tendem a se aproximar dos dados contínuos, enquanto escalas com menos categorias tendem a apresentar maiores problemas de normalidade.

Beal e Dawson (2007) defendendo o uso de escalas de sete pontos argumentam que a partir de determinado ponto, o ganho obtido pelo aumento das opções de resposta apresenta retorno muito reduzido. Desta maneira, optou-se por mensurar os construtos do trabalho através de indicadores ordinais, formados por escalas de sete pontos.

A escala para o construto complexidade ambiental foi construída conforme os elementos que a representam nas definições de Cannon e St. John (2007) e de Sharfman e Dean (1991), ou seja, a heterogeneidade do segmento, sua dispersão, a dificuldade de obtenção de informações e de previsão e entendimento da evolução do segmento. A Figura 2 apresenta os indicadores do construto complexidade ambiental.

\begin{tabular}{|c|c|c|}
\hline FATOR & VARIÁVEL & CONSTRUTO \\
\hline É difícil entender como está evoluindo nosso mercado & com1 & \multirow{8}{*}{ 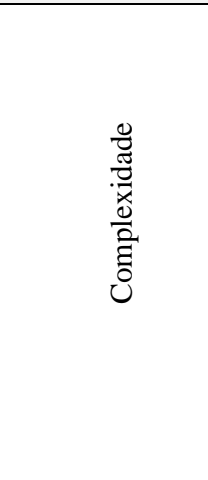 } \\
\hline É difícil obter informações importantes para tomar decisões em nosso segmento & com2 & \\
\hline $\begin{array}{l}\text { É necessário avaliar muitos fatores externos à empresa quando se precisa tomar } \\
\text { uma decisão estratégica }\end{array}$ & $\operatorname{com} 3$ & \\
\hline $\begin{array}{l}\text { Precisamos sempre envidar esforços para entender os fatores externos que } \\
\text { influenciam nosso hotel }\end{array}$ & $\operatorname{com} 4$ & \\
\hline $\begin{array}{l}\text { Em nosso mercado há muitos diferentes tipos de serviços sendo ofertados com } \\
\text { características e preços diferenciados }\end{array}$ & com5 & \\
\hline $\begin{array}{l}\text { Muitas vezes precisamos tomar decisões sem saber que resposta nós receberemos } \\
\text { do mercado (clientes, concorrentes, etc) }\end{array}$ & com6 & \\
\hline $\begin{array}{l}\text { Fatores importantes para a tomada de decisão estão muito dispersos (grande } \\
\text { número de concorrentes, fornecedores e tipos de clientes) }\end{array}$ & $\operatorname{com} 7$ & \\
\hline Tem sido difícil prever as mudanças no nosso segmento & com8 & \\
\hline
\end{tabular}

Figura 2 - Indicadores do construto complexidade ambiental 
A escala para o construto dinamismo ambiental foi construída conforme a proposição conceitual de Lumpkin e Dess (2001) que consideram o dinamismo como a taxa de mudanças imprevistas no ambiente de uma firma. A Figura 3 apresenta os indicadores do construto dinamismo ambiental.

\begin{tabular}{|c|c|c|}
\hline FATOR & VARIÁVEL & CONSTRUTO \\
\hline $\begin{array}{l}\text { As condições econômicas que influenciam nosso mercado têm mudado muito } \\
\text { nos últimos } 3 \text { anos }\end{array}$ & $\operatorname{din} 1$ & \multirow{7}{*}{ 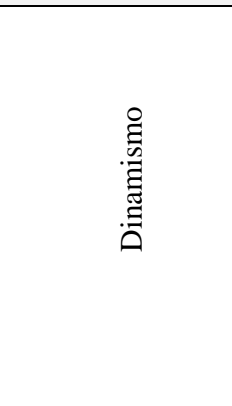 } \\
\hline $\begin{array}{l}\text { As necessidades dos nossos potenciais consumidores têm mudado muito nos } \\
\text { últimos } 3 \text { anos }\end{array}$ & $\operatorname{din} 2$ & \\
\hline A atuação dos nossos concorrentes tem mudado muito nos últimos 3 anos & $\operatorname{din} 3$ & \\
\hline Nossos parceiros e fornecedores têm mudado muito nos últimos 3 anos & $\operatorname{din} 4$ & \\
\hline A regulamentação do setor tem mudado muito nos últimos 3 anos & $\operatorname{din} 5$ & \\
\hline $\begin{array}{l}\text { A tecnologia envolvida com nossas atividades tem mudado muito nos últimos } 3 \\
\text { anos }\end{array}$ & $\operatorname{din} 6$ & \\
\hline $\begin{array}{l}\text { Aspectos sociais e culturais que influenciam nosso mercado têm mudado muito } \\
\text { nos últimos } 3 \text { anos }\end{array}$ & $\operatorname{din} 7$ & \\
\hline
\end{tabular}

Figura 3 - Indicadores do construto dinamismo ambiental

A escala para o construto munificência ambiental foi construída conforme a proposição conceitual de Castrogiovanni (1991), sob a qual a munificência é representada pela capacidade do ambiente, pelo crescimento ou declínio das capacidades do ambiente, e pelo nível de oportunidades ou ameaças existente no ambiente. A Figura 4 apresenta os indicadores do construto munificência ambiental.

\begin{tabular}{|c|c|c|}
\hline FATOR & VARIÁVEL & CONSTRUTO \\
\hline Disputamos um mercado com muitos concorrentes fortes & mun $1^{\mathrm{a}}$ & \multirow{7}{*}{ 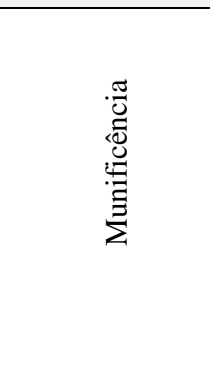 } \\
\hline Nosso mercado tem crescido muito nos últimos 3 anos & mun2 & \\
\hline Estamos em um mercado no qual é muito difícil ser lucrativo & $\operatorname{mun}^{\mathrm{a}}$ & \\
\hline Estamos em um mercado onde há muita rivalidade & $\operatorname{mun} 4^{\mathrm{a}}$ & \\
\hline $\begin{array}{l}\text { As condições gerais do nosso mercado têm melhorado nos últimos } 3 \text { anos } \\
\text { (economia, fornecedores, mão de obra, clientes, recursos) }\end{array}$ & mun5 & \\
\hline $\begin{array}{l}\text { O ambiente empresarial no qual estamos inseridos nos oferece mais } \\
\text { oportunidades do que ameaças }\end{array}$ & mun6 & \\
\hline $\begin{array}{l}\text { Há clientes, fornecedores e outros recursos disponíveis para todos que atuam em } \\
\text { nosso segmento }\end{array}$ & mun7 & \\
\hline
\end{tabular}

Figura 4 - Indicadores do construto munificência ambiental

a Variáveis invertidas, a serem revertidas na tabulação.

Antes da aplicação o instrumento de coleta de dados foi apresentado a dois especialistas da área da hotelaria que aprovaram a adequação do conteúdo e linguagem em relação aos potenciais respondentes.

Para Martins (2006) a validade se refere à capacidade da escala medir o fenômeno, enquanto a confiabilidade se refere à estabilidade dos valores encontrados. A validação da escala foi realizada através da análise fatorial. Segundo Hair et al. (2009) a análise fatorial é usada para analisar inter-relações entre um grande número de variáveis e explicar essas variáveis em termos de fatores comuns, objetivando reduzir o número de variáveis a ser considerado no estudo. Raykov e Marcoulides (2006) explicam que a análise fatorial é ferramenta importante no estudo de variáveis não observáveis, mas hipoteticamente existentes. A redução de variáveis a fatores ajuda a mensurar tais construtos latentes.

Utilizando a Análise Fatorial Exploratória, este trabalho primeiro mensurou a unidimensionalidade dos indicadores utilizados na mensuração de cada construto. Utilizando o software SPSS $®$ 17.0, os indicadores relativos a cada construto passaram pela análise para verificar se todos os indicadores se ajustavam a um único fator.

A avaliação da confiabilidade da escala foi realizada através das análises de comunalidades, da variância extraída pelo fator, do teste de esfericidade de Bartlett, do teste Alfa de Cronbach, das correlações inter itens e item-total e do método split half. Segundo McLeod (2013), o método split half mede a extensão na qual todas as partes da escala contribuem igualmente para a medida. Dois coeficientes são comumente utilizados na avaliação da confiabilidade através do método split half, o Coeficiente de Spearman-Brown e o Coeficiente de Guttman. A Figura 5 apresenta os valores mínimos ideais para cada uma destas medidas. 
Proposição e Teste de uma Escala de Dinamismo, Complexidade e Munificência Ambiental.

\begin{tabular}{|l|c|}
\hline \multicolumn{1}{|c|}{ MEDIDA } & VALOR MÍNIMO ESPERADO \\
\hline Comunalidades & 0,40 \\
\hline Alfa de Cronbach & 0,70 \\
\hline Coeficiente de Spearman-Brown & 0,70 \\
\hline Coeficiente split-half de Guttman & 0,70 \\
\hline Teste de esfericidade de Bartlett & $\mathrm{p}<0,05$ \\
\hline Correlação inter itens & 0,30 \\
\hline Correlação item-total & 0,50 \\
\hline Carga fatorial (para amostras de até $\mathrm{n}=200)$ & 0,40 \\
\hline
\end{tabular}

Figura 5 - Medidas mínimas utilizadas na análise fatorial exploratória.

Fonte: Adaptado de Hair et al. (2009)

Após a avaliação da unidimensionalidade e confiabilidade das escalas, passou-se a rodar a análise fatorial exploratória com os indicadores dos conjuntos de construtos, ou seja, foram realizadas análises com todos os indicadores relacionados às dimensões ambientais. O objetivo desta análise foi identificar quais indicadores apresentavam cargas fatoriais significativas em quais fatores latentes.

Com o objetivo de melhor distinguir os fatores latentes gerados, procedeu-se à rotação dos fatores, através dos métodos Varimax. Hair et al.(2009) afirmam que o objetivo dos métodos de rotação é simplificar a matriz fatorial para melhorar a interpretação, e que o método Varimax se concentra em simplificar as colunas da matriz fatorial garantindo que os fatores extraídos mantenham-se não correlacionados entre si.

\section{RESULTADOS}

$\mathrm{Na}$ apresentação dos resultados antes da avaliação da unidimensionalidade de cada escala proposta, é apresentada a Tabela 1 com a análise fatorial realizada com todos os 22 (vinte e dois) indicadores originais do instrumento. A análise foi realizada pelo método dos componentes principais e a matriz de fatores apresentada é aquela obtida após a rotação Varimax, sendo omitidas as cargas fatoriais inferiores a 0,400 .

Tabela 1 - Matriz rotacionada da análise fatorial inicial com todos os indicadores da escala

\begin{tabular}{|c|c|c|c|c|c|c|}
\hline & \multicolumn{6}{|c|}{ FATORES ROTACIONADOS } \\
\hline & 1 & 2 & 3 & 4 & 5 & 6 \\
\hline com1 & & ,814 & & & & \\
\hline com2 & & ,806 & & & & \\
\hline com3 & & & &, 733 & & \\
\hline com4 & & & & ,785 & & \\
\hline com5 & & & &, 598 & 476 & \\
\hline com6 & & 455 & & & & \\
\hline com7 & & 612 & & & ,496 & \\
\hline com8 & & ,695 & & & & \\
\hline $\operatorname{din} 1$ & 409 & & & & & \\
\hline $\operatorname{din} 2$ & ,759 & & & & & \\
\hline $\operatorname{din} 3$ &, 727 & & & & & \\
\hline $\operatorname{din} 4$ & ,640 & & & & & \\
\hline $\operatorname{din} 5$ & 685 & & & & & \\
\hline $\operatorname{din} 6$ & 674 & & & & & \\
\hline $\operatorname{din} 7$ &, 732 & & & & & \\
\hline mun1 & & & & &,- 735 & \\
\hline mun2 & & & ,755 & & & \\
\hline mun3 & &,- 612 & & & & ,423 \\
\hline mun4 & & & & & & 833 \\
\hline mun5 & & & ,759 & & & \\
\hline mun6 & & & 863 & & & \\
\hline $\operatorname{mun} 7$ & & & 720 & & & \\
\hline Variância explicada & $15,9 \%$ & $14,0 \%$ & $12,2 \%$ & $9,2 \%$ & $7,1 \%$ & $6,2 \%$ \\
\hline
\end{tabular}


Proposição e Teste de uma Escala de Dinamismo, Complexidade e Munificência Ambiental.

Em relação à unidimensionalidade do construto complexidade, a extração de fatores com todos os indicadores originais gerou dois fatores, sugerindo que o construto, medido por tais indicadores, não apresentou unidimensionalidade. Ao apresentar cargas elevadas nos dois fatores extraídos, com cargas maiores no segundo fator do que no primeiro fator, os indicadores "com3", "com4", e "com5", foram excluídos, providência que foi tomada também em relação ao indicador "com6", pois a sua comunalidade extraída foi muito baixa.

Desta maneira, indicadores relacionados à heterogeneidade, como "Com3" e "Com5" que versavam sobre a existência de muitos fatores e de muitas opções no mercado, assim como um indicador relacionado à incerteza de resposta, "Com6", ficaram de fora nos indicadores validados para o construto. Assim, os resultados abaixo apresentados são aqueles obtidos após a exclusão de tais indicadores.

$\mathrm{O}$ teste de esfericidade de Bartlett e a medida de adequação da amostra de Kaiser-Meyer-Olkin foram realizados. A medida de adequação ficou situada em 0,711, dentro do recomendado por Hair et al. (2009) que sugerem 0,700 como ponto de corte para uma medida ideal. Também o teste de Bartlett apresentou significância de 0,000, demonstrando que a matriz de correlações não é uma matriz identidade, e o processo de extração de fatores através do método de componentes principais gerou um fator único, extraído pelo critério de utilização de todos os fatores que apresentem autovalor acima de 1, conseguindo explicar $62 \%$ da variância dos dados. A confiabilidade da escala foi checada pela análise da correlação mínima inter itens, pela análise da correlação mínima item-total, e pela análise do Alfa de Cronbach.

A Tabela 2 apresenta os índices de comunalidade, as cargas no fator extraído, as correlações para cada um dos indicadores de complexidade, bem como a análise do valor de Alfa de Cronbach em caso de exclusões de itens da escala.

Tabela 2 - Índices extraídos para os indicadores de complexidade.

\begin{tabular}{|c|c|c|c|c|c|c|}
\hline $\begin{array}{c}\text { MATRIZ DE } \\
\text { CORRELAÇÕES }\end{array}$ & com1 & $\operatorname{com} 2$ & $\operatorname{com} 7$ & com8 & $\begin{array}{l}\text { CORRELAÇÃO } \\
\text { ITEM-TOTAL }\end{array}$ & $\begin{array}{c}\text { ALFA DE CRONBACH } \\
\text { EXCLUINDO O ITEM }\end{array}$ \\
\hline com1 & 1,000 &, 728 & ,469 & ,446 & 697 & ,701 \\
\hline $\operatorname{com} 2$ & ,728 & 1,000 & 386 &, 501 & 683 & ,709 \\
\hline $\operatorname{com} 7$ & ,469 & ,386 & 1,000 & ,431 &, 510 & ,793 \\
\hline com8 & ,446 &, 501 & 431 & 1,000 &, 554 & ,773 \\
\hline Cargas no fator 1 & 0,855 & 0,847 & 0,702 & 0,742 & & \\
\hline Comunalidades & 0,731 & 0,718 & 0,493 & 0,551 & & \\
\hline \multicolumn{3}{|c|}{ Coeficiente split half de Guttman } & & & 0,722 & \\
\hline \multicolumn{3}{|c|}{ Coeficiente de Spearman-Brown } & & & 0,728 & \\
\hline
\end{tabular}

Vê-se que a menor correlação inter itens apresentada é de 0,386, superior ao mínimo sugerido por Hair et al. (2009) de 0,300. Também a correlação item-total mínima ficou acima do mínimo sugerido de 0,500. O Alfa de Cronbach calculado para a escala foi de 0,798, acima do mínimo sugerido de 0,700, e a análise de possíveis exclusões de itens na escala não apresentou nenhum potencial de melhoria no valor do Alfa de Cronbach.

A primeira análise fatorial realizada sobre os indicadores originais do construto dinamismo demonstrou que apenas o indicador "din1" se mostrou pouco comum aos demais, com um índice de comunalidade de 0,348 e com baixos valores de correlação com os demais indicadores, razão pela qual o indicador foi excluído da análise subsequente.
O teste de Bartlett e a medida de adequação da amostra de Kaiser-Meyer-Olkin ficaram em 0,818 e com significância em 0,000 respectivamente, demonstrando que a matriz de correlações não é uma matriz identidade. O processo de extração de fatores, realizado com o método de componentes principais, gerou um fator único, extraído pelo critério de utilização de todos os fatores que apresentem autovalor acima de 1, que consegue explicar $56 \%$ da variância dos dados. A confiabilidade da escala foi avaliada pela análise das correlações inter itens, correlação item-total e do Alfa de Cronbach calculado.

A Tabela 3 apresenta os índices de comunalidade, as cargas no fator extraído, as correlações para cada um dos indicadores do dinamismo, bem como a análise do valor de Alfa de Cronbach em caso de exclusões de itens da escala. 
Proposição e Teste de uma Escala de Dinamismo, Complexidade e Munificência Ambiental.

Tabela 3 - Índices extraídos para os indicadores do dinamismo

\begin{tabular}{|l|c|c|c|c|c|c|c|c|}
\hline $\begin{array}{c}\text { MATRIZ DE } \\
\text { CORRELAÇÕES }\end{array}$ & $\operatorname{din} 2$ & $\operatorname{din3}$ & $\operatorname{din} 4$ & $\operatorname{din5}$ & $\operatorname{din6}$ & $\operatorname{din} 7$ & $\begin{array}{c}\text { CORRELAÇÃO } \\
\text { ITEM-TOTAL }\end{array}$ & $\begin{array}{c}\text { ALFA DE } \\
\text { CRONBACH } \\
\text { EXLUINDO ITEM }\end{array}$ \\
\hline $\operatorname{din} 2$ & 1,00 &, 661 &, 434 &, 431 &, 485 &, 559 &, 680 &, 807 \\
\hline $\operatorname{din} 3$ &, 661 & 1,00 &, 579 &, 424 &, 370 &, 450 &, 651 &, 811 \\
\hline $\operatorname{din} 4$ &, 434 &, 579 & 1,00 &, 373 &, 360 &, 415 &, 557 &, 830 \\
\hline $\operatorname{din} 5$ &, 431 &, 424 &, 373 & 1,00 &, 553 &, 483 &, 591 &, 824 \\
\hline $\operatorname{din} 6$ &, 485 &, 370 &, 360 &, 553 & 1,00 &, 557 &, 612 &, 819 \\
\hline $\operatorname{din} 7$ &, 559 &, 450 &, 415 &, 483 &, 557 & 1,00 &, 650 &, 811 \\
\hline Cargas fator 1 & 0,802 & 0,778 & 0,693 & 0,719 & 0,736 & 0,773 & & \\
\hline Comunalidade & 0,644 & 0,605 & 0,480 & 0,517 & 0,541 & 0,597 & & \\
\hline Coeficiente split half de Guttman & & & & & & & \\
\hline \multicolumn{2}{l}{ Coeficiente de Spearman-Brown } & & & & & 0,760 \\
\hline
\end{tabular}

A correlação mínima inter itens apresentada foi de 0,360, superior ao mínimo sugerido de 0,300. A correlação item-total mínima foi de 0,557 , superior ao mínimo sugerido de 0,500, o Alfa de Cronbach calculado foi de 0,843 , e na análise de exclusões simuladas de itens da escala, nenhuma exclusão teve potencial para elevar o valor do Alfa calculado.

A análise inicial dos indicadores originais do construto munificência demonstrou que os indicadores "mun1", "mun3", e "mun4", relacionados à disputa em um mercado com muitos concorrentes fortes, à dificuldade de obtenção de lucratividade e à existência de rivalidade, respectivamente, apresentaram baixa correlação com os demais indicadores, além de terem obtido cargas elevadas em um fator diferente dos demais indicadores.

As afirmações representadas por tais indicadores foram dispostas de maneira reversa, ou seja, valores elevados representariam baixa munificência enquanto valores baixos representariam elevada munificência. É possível que esta situação tenha conduzido os respondentes a algum viés, assim, tais indicadores foram excluídos da análise subsequente.
Por outro lado, o trabalho de Wang e Ang (2004) considerou a intensidade de competição e a própria rivalidade como componentes de outra dimensão que ele chamou de hostilidade, desta forma, também é possível que os indicadores aqui excluídos façam parte desta outra possível dimensão do ambiente.

$\mathrm{Na}$ análise fatorial seguinte, o teste de Bartlett e a medida de adequação da amostra de Kaiser-MeyerOlkin ficaram em 0,754 e com significância em 0,000 respectivamente, demonstrando que a matriz de correlações não é uma matriz identidade. O processo de extração de fatores, realizado através do método de componentes principais gerou um fator único extraído, derivado de um autovalor maior do que 1, explicando $63 \%$ da variância. A confiabilidade da escala foi avaliada pela análise das correlações inter itens e itemtotal, além da análise do valor do Alfa de Cronbach calculado para a escala.

A Tabela 4 apresenta os índices de comunalidade, as cargas no fator extraído, as correlações para cada um dos indicadores de munificência, bem como a análise do valor de Alfa de Cronbach em caso de exclusões de itens da escala.

Tabela 4 -Índices extraídos para os indicadores de munificência

\begin{tabular}{|l|c|c|c|c|c|c|}
\hline $\begin{array}{c}\text { MATRIZ DE } \\
\text { CORRELAÇÕES }\end{array}$ & mun2 & mun5 & mun6 & mun7 & $\begin{array}{c}\text { CORRELAÇão } \\
\text { ITEM-TOTAL }\end{array}$ & $\begin{array}{c}\text { ALFA DE CRONBACH } \\
\text { EXCLUINDO O ITEM }\end{array}$ \\
\hline mun2 & 1,000 &, 531 &, 603 &, 423 &, 636 &, 740 \\
\hline mun5 &, 531 & 1,000 &, 625 &, 322 &, 591 &, 760 \\
\hline mun6 &, 603 &, 625 & 1,000 &, 531 &, 746 &, 683 \\
\hline mun7 &, 423 &, 322 &, 531 & 1,000 &, 498 &, 809 \\
\hline Cargas no fator 1 & 0,810 & 0,786 & 0,880 & 0,694 & & \\
\hline Comunalidade & 0,657 & 0,618 & 0,775 & 0,482 & & \\
\hline
\end{tabular}


Proposição e Teste de uma Escala de Dinamismo, Complexidade e Munificência Ambiental.

A mínima correlação inter itens apresentada foi de 0,322, valor superior ao mínimo sugerido de 0,300. Percebe-se também que o valor da correlação item-total mínimo apresentado foi de 0,591 , superior ao mínimo sugerido de 0,500. O Alfa de Cronbach calculado para a escala ficou em 0,800 , superior ao mínimo sugerido de 0,700. A análise das exclusões simuladas de itens da escala não produziu grandes diferenças no valor do Alfa calculado. Apenas a exclusão do indicador "mun7" apresentou um potencial de aumentar levemente o valor do Alfa de Cronbach, mas não o suficiente para exigir qualquer providência.

\subsection{Validação Conjunta das Escalas}

Uma vez checada a unidimensionalidade dos construtos e seus indicadores, procurou-se avaliar como se comportam tais indicadores quando analisados em conjunto com os indicadores dos demais construtos. A seguir são apresentados os resultados da análise fatorial realizada com todos os indicadores dos construtos complexidade, dinamismo e munificência. $\mathrm{O}$ objetivo desta análise é verificar quantos e quais fatores são criados na análise, e quais indicadores efetivamente pertencem a quais construtos.

$O$ processo de extração de fatores, realizado através do método de componentes principais, utilizando o critério de validar fatores com um autovalor maior do que 1 gerou três fatores, que juntos explicam $61,7 \%$ da variância.

A Tabela 5 apresenta os três fatores extraídos com as cargas obtidas por cada indicador em cada fator. No lado esquerdo da tabela constam as cargas da análise fatorial original sem rotação, enquanto no lado direito são apresentadas as cargas após a rotação Varimax, ocultas as cargas inferiores a 0,400.

Tabela 5 - Fatores e cargas dos construtos das dimensões ambientais.

\begin{tabular}{|c|c|c|c|c|c|c|}
\hline & \multicolumn{3}{|c|}{$\begin{array}{l}\text { FATORES ORIGINAIS SEM } \\
\text { ROTAÇÃO } \\
\end{array}$} & \multicolumn{3}{|c|}{ FATORES APÓS ROTAÇÃO VARIMAX } \\
\hline & $\mathbf{1}$ & 2 & 3 & Dinamismo & Munificência & Complexidade \\
\hline com1 &, 578 &,- 322 &, 562 & & &, 842 \\
\hline com2 &, 532 &,- 426 &, 503 & & & ,837 \\
\hline com7 & ,490 &,- 419 & ,261 & & & ,650 \\
\hline com8 & ,483 &,- 499 & ,306 & & &, 721 \\
\hline $\operatorname{din} 2$ &, 707 &,- 019 &,- 388 & ,796 & & \\
\hline $\operatorname{din} 3$ & ,674 &,- 043 &,- 408 &, 783 & & \\
\hline $\operatorname{din} 4$ & ,651 &,- 243 &,- 232 & ,664 & & \\
\hline $\operatorname{din} 5$ & ,687 & ,008 &,- 175 & ,648 & & \\
\hline $\operatorname{din} 6$ & ,691 &, 146 &,- 266 & ,702 & & \\
\hline $\operatorname{din} 7$ &, 701 &,- 048 &,- 308 &, 743 & & \\
\hline mun2 & ,465 & ,647 & ,116 & &, 757 & \\
\hline mun5 &, 327 &, 731 &, 089 & & ,766 & \\
\hline mun6 &, 453 & ,684 &, 326 & &, 871 & \\
\hline mun7 & ,264 &, 520 &, 458 & & ,720 & \\
\hline
\end{tabular}

Considerando a situação de que a simples análise fatorial produziu três fatores não bem distintos, com a presença de cargas cruzadas e fatores contendo cargas positivas e negativas, sendo necessária melhor explicação dos fatores, optou-se por realizar a análise com rotação Varimax. O lado direito da tabela, demonstra que a rotação Varimax foi capaz de gerar três fatores bem distintos e que estes fatores tiveram bom ajuste com os indicadores teorizados. O fator 1 extraído pode ser chamado de "Dinamismo", enquanto o fator 2 pode ser chamado de "Munificência", e o fator 3 de "Complexidade".

\section{DISCUSSÃO DOS RESULTADOS}

Os indicadores finais dos fatores extraídos na análise fatorial apresentam coerência com a proposta de Lukas, Tan e Hult (2001), segundo a qual a complexidade e o dinamismo representam o ambiente como fonte de informações para a firma, enquanto a munificência representa o ambiente como fonte de recursos.

Em relação à escala de mensuração da complexidade, os indicadores que apresentaram bom ajuste ao construto são aqueles relacionados à dificuldade de obtenção de informações, dificuldade de 
Proposição e Teste de uma Escala de Dinamismo, Complexidade e Munificência Ambiental.

entendimento, dispersão, e imprevisibilidade do ambiente. Os indicadores relacionados à heterogeneidade usados originalmente não obtiveram ajuste ao construto, apresentando-se como multidimensionais. Talvez, como já preconizava
Khandwalla (1972), a heterogeneidade seja uma dimensão distinta do ambiente.

A Figura 6 apresenta os indicadores validados relativos ao construto complexidade ambiental.

\begin{tabular}{|l|c|}
\hline \multicolumn{1}{|c|}{ FATOR } & VARIÁVEL \\
\hline É difícil entender como está evoluindo nosso mercado & com1 \\
\hline É difícil obter informações importantes para tomar decisões em nosso segmento & com2 \\
\hline $\begin{array}{l}\text { Fatores importantes para a tomada de decisão estão muito dispersos (grande número de concorrentes, } \\
\text { fornecedores e tipos de clientes) }\end{array}$ & com7 \\
\hline Tem sido difícil prever as mudanças no nosso segmento & com8 \\
\hline
\end{tabular}

Figura 6 - Indicadores validados para o construto complexidade ambiental

A imprevisibilidade do ambiente, como proposta por Lukas, Tan e Hult (2001) se apresentou como componente do construto complexidade, diferente do que haviam proposto Lumpkin e Dess (2001) de que a imprevisibilidade das mudanças seria um componente do dinamismo. A existência de mudança sinaliza para o dinamismo, mas a imprevisibilidade das mudanças está mais relacionada ao construto complexidade.

Entre os indicadores propostos para o construto dinamismo, o único indicador que não apresentou ajuste suficiente foi aquele relacionado às mudanças nas condições econômicas. As mudanças nas necessidades dos consumidores, na atuação dos concorrentes, nos fornecedores e parceiros, na regulamentação do setor, na tecnologia e nos aspectos sociais se apresentaram como unidimensionais no construto dinamismo. Isto está coerente com o proposto por Harrigton e Kendall (2007), destoando da proposta de Luo, Tan e O’Connor (2001) de que a imprevisibilidade faria parte do construto. A Figura 7 apresenta os indicadores que foram validados para o construto dinamismo.

\begin{tabular}{|l|c|}
\hline \multicolumn{1}{|c|}{ INDICADOR } & VARIÁVEL \\
\hline As necessidades dos nossos potenciais consumidores têm mudado muito nos últimos 3 anos & din2 \\
\hline A atuação dos nossos concorrentes tem mudado muito nos últimos 3 anos & din3 \\
\hline Nossos parceiros e fornecedores têm mudado muito nos últimos 3 anos & din5 \\
\hline A regulamentação do setor tem mudado muito nos últimos 3 anos & din6 \\
\hline A tecnologia envolvida com nossas atividades tem mudado muito nos últimos 3 anos & din7 \\
\hline Aspectos sociais e culturais que influenciam nosso mercado têm mudado muito nos últimos 3 anos & \\
\hline
\end{tabular}

Figura 7 - Indicadores validados para o construto dinamismo ambiental

Assim, a escala de mensuração do construto dinamismo se apresentou como unidimensional e composta pela variabilidade dos diversos componentes do ambiente competitivo da firma, porém não relacionada à imprevisibilidade destas mudanças.

De maneira geral, os indicadores do construto munificência que foram validados neste trabalho estão relacionados à existência de recursos disponíveis para todos os competidores no ambiente, à oferta maior de oportunidades do que de ameaças no ambiente, à melhoria das condições e ao crescimento do mercado. Os indicadores relacionados à dificuldade da lucratividade, à rivalidade, e à existência de muitos concorrentes fortes no mercado, usados de maneira reversa neste estudo, não se ajustaram ao fator validado. É possível que estes indicadores façam parte de outro construto, chamado por Wang e Ang (2004) de hostilidade.

A Figura 8 apresenta os indicadores que foram validados para o construto munificência.

\begin{tabular}{|l|c|}
\hline \multicolumn{1}{|c|}{ FATOR } & VARIÁ VEL \\
\hline Nosso mercado tem crescido muito nos últimos 3 anos & mun2 \\
\hline $\begin{array}{l}\text { As condições gerais do nosso mercado têm melhorado nos últimos 3 anos (economia, } \\
\text { fornecedores, mão de obra, clientes, recursos) }\end{array}$ & mun5 \\
\hline O ambiente empresarial no qual estamos inseridos nos oferece mais oportunidades do que ameaças & mun6 \\
\hline Há clientes, fornecedores e outros recursos disponíveis para todos que atuam em nosso segmento & mun7 \\
\hline
\end{tabular}

Figura 8 - Indicadores do construto munificência ambiental 
Proposição e Teste de uma Escala de Dinamismo, Complexidade e Munificência Ambiental.

Portanto, este trabalho valida a proposta de Castrogiovanni (1991), segundo a qual a munificência é composta pela capacidade do ambiente, pelo crescimento ou declínio das capacidades do ambiente, e pelo nível de oportunidades ou ameaças existente no ambiente.

\section{CONSIDERAÇÕES FINAIS}

A proposição de novas escalas para os construtos relacionados às dimensões ambientais pode ser creditada como um avanço deste trabalho, principalmente por propor escala adaptada para a mensuração da percepção do gestor quanto aos aspectos elencados pela teoria como componentes de cada construto.

Os resultados da análise dos indicadores das escalas demonstram haver multidimensionalidade entre os construtos estudados, ou seja, dinamismo, complexidade e munificência podem ser estudados independentemente uns dos outros e, seus efeitos sobre a estratégia e sobre o desempenho das firmas são independentes entre si.

O construto complexidade, pelos indicadores que foram validados neste trabalho, pode ser visto como formado pela dificuldade de obtenção de informações e de entendimento do ambiente, pela dispersão da informação no ambiente e pela sua imprevisibilidade.

O construto dinamismo pode ser entendido como a variabilidade nos diversos componentes do ambiente competitivo no qual a firma está inserida. Componentes relacionados a consumidores, concorrentes, fornecedores, aspectos sociais, tecnologia, e regulamentação do setor, devem ser considerados quando se visa mensurar o dinamismo de um ambiente competitivo.

O construto munificência se caracterizou como a existência de recursos disponíveis a todos os competidores, a ampliação da disponibilidade de recursos, a oferta de mais oportunidades do que ameaças aos competidores e o crescimento do mercado. Indicadores relacionados à competição e rivalidade não apresentaram ajuste nesta dimensão, sendo importante a realização de novos estudos no sentido de verificar uma possível existência de outra dimensão relacionada à hostilidade.

Os construtos complexidade e dinamismo fazem parte de um contexto que considera o ambiente como fonte de informações para o gestor, enquanto o construto munificência faz parte de um contexto no qual o ambiente é visto como fonte de recursos para exploração por parte da firma. Talvez isto explique o fato de que nem sempre os três construtos têm sido estudados em conjunto, havendo uma maior predominância de estudos conjuntos do dinamismo e da complexidade, enquanto os estudos sobre a munificência muitas vezes utilizam apenas este construto.

Este trabalho oferece contribuição importante ao disponibilizar escalas válidas para novos estudos que procurem relacionar os construtos das dimensões ambientais a outros construtos estudados, permitindo testes de relacionamentos entre construtos, como abordagens de equações estruturais. Como o trabalho foi aplicado com uma amostra de gestores da indústria hoteleira, seria interessante adotar a mesma abordagem em outro segmento com o intuito de avaliar a estabilidade e aplicabilidade da escala a outras realidades.

Assim, sugere-se que outras formas de validação das escalas sejam testadas, inclusive em outros setores empresariais, o que contribuiria para o refinamento das mesmas, tornando produtivo o seu uso em um amplo número de estudos hipotéticos.

\section{REFERÊNCIAS}

Andrews, R. (2009). Organizational task environments and performance: an empirical analysis. International Public Management Journal, 12(1), $1-23$.

Andrews, R., \& Johansen, M. (2012). Organizational environments and performance: a linear or nonlinear relationship? Public Organization Review, 12, 175-189.

Beal, D J., \& Dawson, J. F. (2007). On the use of Likert-Type scales in multilevel data: influence on aggregate variables. Organizational Research Method, 10(4), 657-672.

Bertucci, J. L. de O. (2005). Ambiente, estratégia e performance organizacional no setor industrial e de serviços. Revista de Administração de Empresas, 45(3), 10-24.

Bollen, K. A. (1989). Structural equation modeling with latent variables. New York: Wiley \& Sons.

Boyd, B. K., Dess, G. G., \& Rasheed, A. M. A. (1993). Divergence between perceptual and archival measures of the environment: causes and consequences. Academy of Management Review, 18, 204-226.

Cannon, A. R., \& St. John, C. H. (2007). Measuring environmental complexity: a theoretical and empirical assessment. Organizational Research Methods, 10(2), 296-321.

Castrogiovanni, G. J. (1991). Environmental munificence: a theoretical assessment. Academy of Management Review, 16(3), 542-565. 
Proposição e Teste de uma Escala de Dinamismo, Complexidade e Munificência Ambiental.

Child, J. (1972). Organizational structure, environment and performance: the role of strategic choice. Sociology, 6, 1-22.

Davis, J. P., Eisenhardt, K. M., \& Bingham, C. B. (2009). Optimal structure, market dynamism, and the strategy of simple rules. Administrative Science Quarterly. 54, 413 - 452.

Dess, G. G., \& Beard, D. W. (1984). Dimensions of organizational task environments. Administrative Science Quarterly, 29, 52-73.

Duncan, R. B. (1972). Characteristics of organizational environments and perceived environmental uncertainty. Administrative Science Quarterly, 313327.

Finney, S. J., \& Distefano, C. (2006). Non-normal and categorical data in structural equation modeling. In Hancok, G.R., \& Mueller, R. O. Structural Equation Modeling: A Second Course. Charlotte, NC: Information Age Publishing.

Gardelin, J. P. (2010). O Comportamento estratégico e a percepção de incerteza e munificência de micro e pequenas empresas. Dissertação de mestrado, Mestrado em Administração, Universidade do Vale do Itajaí, Biguaçu/SC.

Hair Jr, J. F., Black, W. C., Babin, B. J., Anderson, R. E., \& Tatham, R. L. (2009). Análise multivariada de dados (5a ed.). Porto Alegre: Bookman.

Harrington, R. J., \& Kendall, K. W. (2005). How certain are you measuring environmental dynamism and complexity? A multitrait-multimethod approach. Journal of Hospitality \& Tourism Research, 29, 245-275.

Harrington, R. J., \& Kendall, K. W. (2007). Uncovering the interrelationships among firm size, organizational involvement, environmental uncertainty, and implementation success. International Journal of Hospitality \& Tourism Administration, 8(2), 1-24.

Harris, R. D. (2004). Organizational task environments: an evaluation of convergent and discriminant validity. Journal of Management Studies, 41, 857-882.

Jogaratnan, G., Tse, E. C., \& Olsen, M. D. (1999). An empirical analisys of entrepreneurship and performance in the restaurant industry. Journal of Hospitality \& Tourism Research, 23(4), 339-353.

Jogaratnan, G. (2002). Entrepreneurial orientation and environmental hostility: an assessment of small, independent restaurant businesses. Journal of Hospitality \& Tourism Research, 26, 258-277.

Khandwalla, Pr. N. (1972). Environment and its impact on the organization. International Studies of Management \& Organization, 2(3), 297-313.

Lombardi, M. F. S., \& Brito, E. P. Z. (2010). Incerteza subjetiva no processo de decisão estratégica: uma proposta de mensuração. Revista de Administração Contemporânea, 14(6), 990-1010.

Lumpkin, G. T., \& Dess, G. G. (2001). Linking two dimensions of entrepreneurial orientation to firm performance: the moderating role of environment and industry life cycle. Journal of Business Venturing, 16, 429-451.

Luo, Y., Tan, J.J., \& O’Connor, N. G. (2001). Strategic response to a volative environment: the case of cross-cultural cooperative ventures. Asia Pacific Journal of Managemen, 18, 7-25.

Malgueiro, A. R. (2011). A relação entre inovação, ambiente e desempenho: um estudo nas empresas instaladas em incubadoras tecnológicas de Santa Catarina. Dissertação de mestrado, Mestrado em Administração, Universidade do Vale do Itajaí, Biguaçu/SC.

Martins, G. A. (2006). Sobre confiabilidade e validade. RBGN. 8(20), 1-12.

McLeod, S. (2013). What is reliability? Disponível em: www.simplypsychology.org/reliability.html

Milliken, F. J. (1987). Three types of perceived uncertainty about the environment: state, effect, and response uncertainty. Academy of Management Review, 12, 133-143.

Nadkarni, S., \& Barr, P. S. (2008). Environmental context, managerial cognition, and strategic action: an integrated action. Strategic Management Journal, 29, 1395-1427.

Olsen, M. D. (2004). Literature in strategic management in the hospitality industry. Hospitality Management, 23, 411-424.

Osborn, R. N., \& Hunt, J. G. (1974). Environment and organizational effectiveness. Administrative Science Quarterly, 19, 231-246.

Pfeffer, J., \& Salancik, G. R. (1978). The external control of organizations: a resource dependence perspective. New York: Harper \& Row. 
Porto, É. C., Brito, L. A. L., Silva, A. A., Bataglia, W., \& Brito, E. Z. (2009). Ambientes organizacionais: uma proposta de classificação com uso de munificência, dinamismo e complexidade. Base, 6(2), 101-119.

Qu, W. G., Pinsonneault, A., \& Oh, W. (2011). Influence of industry characteristics on information technology outsourcing. Journal of Management Information Systems, 27(4), 99-127.

Rossetto, C. R., Rossetto, A. M., Verdinelli, M. A., \& Carvalho C. E. (2012). Novas evidências na relação ambiente organizacional, comportamento estratégico e desempenho: um estudo multi-caso em agências de viagens da grande Florianópolis (SC). Revista Turismo \& Desenvolvimento (Online), 2, $717-728$.

Salazar, A. J., \& Peláez, E. (2011). The organic growth of dynamics capabilities for innovation within resource constrained environments. International Journal of Technology Management \& Sustainable Development. 10 (3), 231 - 250.

Sharfman, M. P., \& Dean Jr., J. W. (1991). Conceptualizing and measuring the organizational environment: a multidimensional approach. Journal of Management, 17(4), 681-700.

Simsek, Z., Veiga, J. F., \& Lubatkin, M. H. (2007). The impact of managerial environmental perceptions on corporate entrepreneurship: towards understanding discretionary slack's pivotal role.
Journal of Management Studies, 44(8), 1398 1424.

Tan, J., \& Litschert, R. J. (1994). Environment-strategy relationship and its performance implications: an empirical study of the Chinese electronics industry. Strategic Management Journal, 15, 1-20.

Tan, J. (2002). Impact of ownership type on environment-strategy linkage and performance: evidence from a transitional economy. Journal of Management Studies, 39(3), 333-354.

Tan, J., \& Tan, D. (2005). Environment-strategy coevolution and co-alignment: a staged model of Chinese SOEs under transition. Strategic Management Journal, 26, 141-157.

Teixeira, O. R. de P., Rossetto, C. R., \& Carvalho, C. E. (2009). O relacionamento entre o ambiente organizacional e o comportamento estratégico: um estudo no setor hoteleiro de Florianópolis - SC. Turismo: Visão e Ação, 11(2), 157-174.

Thompson, J. D. (1967). Organization in action. New York: McGraw-Hill.

Wang, C. K., \& Ang, B. L. (2004). Determinants of venture performance in Singapore. Journal of Small Business Management. 42 (4), 347 - 363.

Xue, L., Ray, G., \& Sambamurthy, V. (2012) Efficiency or innovation: how do industry environments moderate the effects of firms it asset portfolios?MIS Quarterly, 36(2), 509 - 528. 